\title{
Discussion on the Simplicity in Modern Product Design Junying Shi ${ }^{1}$ and Jun Chen ${ }^{2}$
}

(Hubei Institute of Engineering, Xiaogan, Hubei 432000)

\begin{abstract}
With the progress of Science and technology, people's lives are more convenient, but also faced with some new problems: the city's pollution, complicated work and stressful life. So people began to look forward to simple and natural style life; abandon the harassment and unnecessary luxury, to seek a natural peace of mind. The simple design of the product modeling language just meets the needs of the people. This article elaborate the combination of the simplicity design concept and product aesthetics, design the product with harmony life and more fashionable characteristics.
\end{abstract}

Keywords: Product design; Modeling language; Simplicity

\section{浅析现代产品设计中的简约性}

\author{
师俊英 ${ }^{1}$ 陈军 \\ (湖北工程学院，湖北 孝感 432000)
}

摘要: 科技在进步, 人们的生活也更加便利, 但也面临着一些新生的问题: 城市的污染, 繁杂的工作和紧张的生 活压力。因此人们开始向往简单自然的简约风格; 摒弃繁㻺和无谓的豪华, 寻求一种自然平实的心境。产品造型语 言的简约设计恰好满足人们需求。本文讲述简约设计理念和产品美学结合, 设计出与生活和谐统一, 更具时代感的 产品。

关键词：产品设计；造型语言；简约

中图分类号: K825.72 文摘标识码: A

引言

在我们生活的周围, 每时每刻都会看到或接触到玲珑满目的工业产品。而且现代产品的造型也 在不断的创新和更换, 与人们日益增长的物质生活和精神生活的需求相匹配。产品如果想要在达到 完美展现, 它的造型设计至关重要。产品的造型设计要与时代发展相结合, 同时需要蕴涵艺术的美 感, 不但提高人们的生活质量也满足人们对艺术精神层次的追求。

\section{1 产品造型的概念及其现状}

产品的造型设计就是把产品的实用性和艺术性完美结合, 进行合理的规划创造出好的产品。产 品造型主要目的是为了体现出产品的使用价值, 能更好的为人们所使用, 给人们的日常生活带来便 利。另外一个目的是体现出产品的审美艺术, 产品需要有其特定的结构和外观形态。产品结构与形 态的合理, 才能使产品的使用功能得以展现, 同时也需要产品具备造型的美感。不同功能的产品要 求不同的造型, 即使一样功能要求的产品也需要有不同的造型。这就需要有一定的设计原则

产品造型的设计原则：实用、经济、美观。具体来讲实用主要指产品的使用方便与性能好。对 于使用者来说, 还要有操作方便、简单易上手等特点。经济就是产品的价格便宜, 成本低, 原材料 耗费少, 制作的人力需求少。美观是表现人们对产品的造型的喜爱, 符合美学规律和人们的审美观 念。三个原则需要合理的结合起来, 按这样造型原则设计出来的产品才会是符合人们生活需求的。

现代产品造型设计俨然成为我们日常生活中息息相关的一部分，其中能体现人们基本生活需求 
和发展需要。而产品造型设计的问题, 与工业生产力息息相关, 在技术、质量、性能、功能等方面 发展变化。现今产品越来越重视造型设计感的成分, 产品最终是为了服务消费者, 如果是一种商品, 那么商品就应该满足人们的生活需要, 当今商品竞争, 看起来是物品的比较, 实际上是设计和技术 相互结合的比拼, 尤其是设计。只有创造出优秀的产品, 设计出精美的产品造型, 才能更好的赢得市 场。

畅销的产品是主要由它的设计决定的。产品的水平优劣也与工业生产力的水平高低息息相关, 现在工业化的发展也越来越成熟, 各种类别的产品也有各自的发展和演化。在这些造型设计中成熟 的系统经验应该得以保持加以利用。同时也要创造出符合时代气息的创新点。

总之, 现代产品造型设计既要遵循工业技术能力, 又要遵循艺术创造内涵的前提下, 努力通过设 计来发展和提高, 改善人民的文化生活, 提高人们生活和工作的质量。

\section{2 产品简约性的特点及具体表现}

简约而不简单, 人们在注重产品需要的使用价值以外, 开始越来越重视产品所表现的美感内涵。 而简约设计彰显出产品独特的美感，所以简约的产品受到越来越多人们的喜爱。简约原本是表现一 种单纯明快, 修辞简省而语意丰富的文体风格。之后又慢慢地发展延伸为一种设计风格。这种风格 一经产生就在设计领域得到了广泛的发展, 成为当今一种主流的设计风格。

简约设计的特点, 简约并不代表着简单和单调。简约是经过不断把好的方面组合和精选来力求 完美, 往往能达到以少胜多的效果。在简约设计中, 简约是指对于产品设计的一种精简的概括。在 细节上追求细致入微, 抓住关键的因素和条件进行认真的概括。找出最适合的设计方法, 减少整体 框架的累赘，以简明自然的方法达到最佳的表现。

现今，简约设计逐步成为主流的设计风格，已经深入影响到建筑家居、产品设计、平面广告等 许多的领域。

简约设计以简明自然的方法达到最佳最丰富的表现，来满足人们对于生活质量的需求。现代人 们的生活节奏越来越快, 生活负荷无时无刻影响着人们的生活情调。人们渴望在这繁忙沉重的生活 中得到一种放松。这就影响着人们越来越追求简单和自然的心理需要。简约设计恰当的满足了人们 的这种需求, 实质也是人们的这种需求才产生了简约设计。

简约设计反映了人们对于自然和轻松的渴望, 贴合了现代人对生活的价值观念。同时现代工业 化的发展, 环境的破坏和资源的紧缺和浪费等一系列问题, 也在深远的影响着人们的生活。简约的 设计恰当的体现了这样的条件, 这也是简约产品必然发展和适于时代发展的因素。

简约并不意味着简单乏味, 简约的设计具有自身的特性, 蕴含简单、纯粹的想象力和创造性。 简约的产品是更具时代特征的美感，让人们的生活更加简单舒适。

\section{1 产品功能的简约}

产品功能的简约体现出产品的内容美也就是实用性。现今, 产品功能设计似乎走入了一种误区, 只要功能越多产品价值就越高产品越好。但是一个产品功能越多, 必然的产品结构便会更加复杂, 进一步影响到产品的实用性能, 而且这些附加的功能, 更多地是起到一些 “装点门面” 性质的作用。 有的甚至会影响到主要功能的运作, 其次附加的功能, 必然会增加产品的价格。不断的向产品中增 
加功能, 是不可持续发展的方向。

譬如现在的手机产品, 最初概念是：可携带电话机。然而通话这个主要的功能却越来不被重视, 又或者是湮没在附加功能中了, 现在一款新手机的推出, 通常的噱头都是增加了什么样的新功能。手 机仿佛得改称为 “万能机” 。可这样一部 “万能机” , 人们在购买之后真正能够用起来的功能又有多 少呢? 还要为这些多余的功能买单, 又或者在这些杂乱的功能中摸索得以运用。

荷兰的设计公司 “John” s Phone” 设计开发了一款 “反其道而行” 的手机（如图 4）。这款手机 只有一个功能: 打电话! 就连配置的耳机都不能听音乐, 却能用来通话。如今, 人们购买消费的时 候越来越注重产品的性价比。这是一个产品要想取得成功的一个新的思考点和方向。

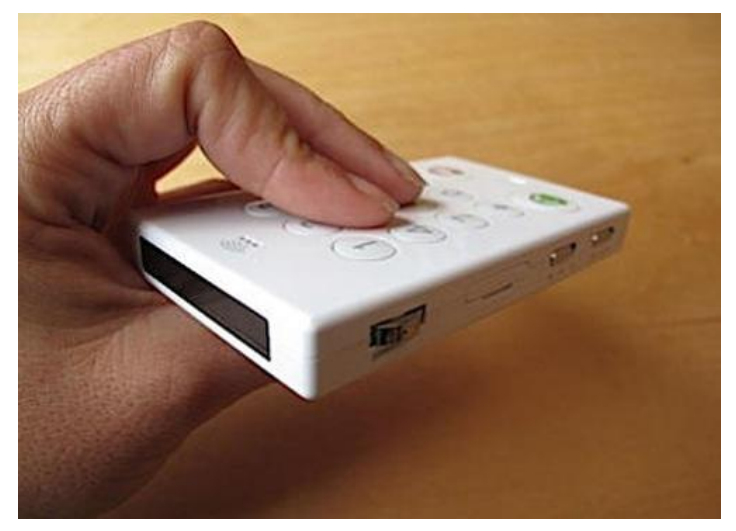

图 4 John's Phone

功能的简约不是产品功能性的缺乏单一, 而是产品使用性质上的专业, 而不是用杂乱的功能拼凑 在一起, 却相互制约。在产品造型设计中, 把功能加强和提炼, 减少不切实用的功能, 才是产品功能 的简约美。

\section{2 产品形态的简约}

产品形态的简约展示出产品的体积美。简洁、干练的形态使产品更具有新时代的表现力。

在一个产品的形态中, 形态表现得越简练, 产品的制作工艺就越便利, 形态的复杂不仅仅会影响 到产品的生产制作, 还会不自然地增加产品使用的复杂程度, 复杂的操作过程往往会使人厌烦。产品 形态以最简、最优的表现, 让人感到便捷与舒适, 才更加具有时代性的美感。

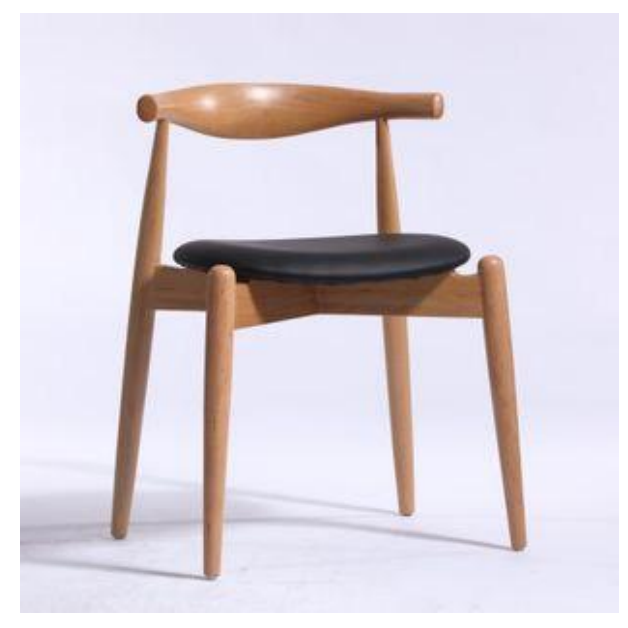

图 5 牛角椅

用极少的形式去简化形态, 以最合理的形态去承担功能, 来自北欧的设计师设计的牛角椅 (如图 $5)$, 简约的形态, 通过简单的操作, 使椅子具有多重的坐姿享受。而且以如此简约的形态的呈现, 使椅 
子具有的它独特的美感。

形态的简约不是缩减产品体积的结构, 而是摒弃不必要的东西。在产品造型设计中, 把形态通过 简单合理的组合, 在细节中追求完美。表现出一种质朴自然, 同时蕴涵少极是多的表现力, 才是产 品形态的简约美。

\section{3 产品色彩的简约}

产品色彩的简约展示出产品的外在美。干净、轻松的色彩搭配使产品更具有特有韵味。产品繁 琐花哨的色彩不是一种好的表现形式, 尤其是在现在这种快速的生活节奏中, 一个简洁的色彩表现能 给人们带来舒服, 自然的感受, 一些复杂繁琐, 不逻辑化繁杂的色彩搭配, 往往会让人感觉到俗气和花 梢。

产品色彩的美感的体现, 主要是贴合人们的心理需求。色彩的美感需要色彩适当的调和和搭配。 这是苹果 1998 年推出的 iMac (如图 6)。产品用红、橙、蓝、绿、紫与大面具的白色简单的结合起来, 合理的搭配组合令人眼前一亮。特别是彩色机壳半透明颜色渐变, 想象力十足。

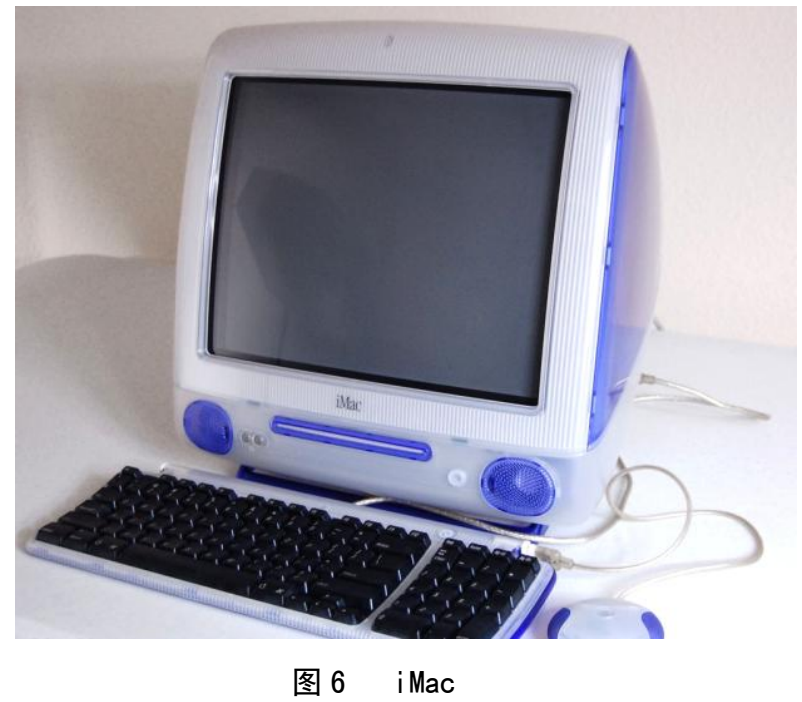

色彩的简约不是产品色彩的单调。相反地用简洁的色彩进行合理的组合和搭配, 会让人眼前一 亮, 让人百看不厌, 才是产品色彩的简约美。

\section{3 结束语}

本文阐述了产品造型设计中简约风格的体现, 并分析了产品造型设计未来发展的一些探索和思 考。产品造型设计越来越系统, 也产生了更宽泛的方向和复杂的问题。认清这些方向和解决这些问 题, 需要更多地去贴近人们日常生活的生活需要, 真正做到以人们的需求来设计, 而不是以自我的 理解设计产品, 要站在使用者的角度和观念去思考。让产品造型设计更贴近人们的需求的本质, 这 是产品造型设计的出发点和着重点。

简约的产品不意味的简单。简约的产品具有自身的特性和个性。随着社会的发展, 资源环境的 的破坏和浪费以及人们精神水平得提高。人们逐渐唤醒加强了对于环境保护的意识, 这就需要人们 在使用需求上更加的精选化, 产品的设计更加的实用, 简约的设计体现了这样的条件, 这也是简约 产品必然发展和适于时代发展的因素。 


\section{参考文献}

$[1]$ 兰海龙. 禅学思想在产品设计中的应用 $[J]$. 郑州轻工业学院学报: 社会科学版, 2013

[2] 李寅. 浅析斯堪的纳维亚现代玻璃设计发展 [J]. 大众文艺: 学术版, 2013

[3]陈庆. 简约而不简单 $[\mathrm{J}]$. 学生之友, 2011

[4]卢冬冬. 论细节意识在产品设计中的重要性 [J].大众文艺, 2014

[5]龚飞风. 梁志天的简约主义设计风格 $[\mathrm{J}]$. 艺海, 2013

\section{作者简介：}

师俊英 (1981.6-) 男, 山西临汾人, 湖北工程学院讲师, 硕士研究生, 研究方向: 产品设计

陈 军 (1979. 8-) 男, 湖北公安人, 博士研究生, 研究方向: 设计学

\section{References}

[1] Lan Hailong. The application of Zen thought in product design [J]. Journal of Zhengzhou University of Light Industry: Social Science Edition, 2013

[2] Li Yin. Analysis on the development of Scandinavian modern glass design [J]. Popular Arts: academic version, 2013

[3] Chen Qing. Concise but not simple [J]. Friends of the Students, 2011

[4] Lu Dongdong. Discussion on the importance of detail awareness in product design [J]. Popular Literature, 2014

[5] Gong Feifeng. Liang Zhitian's simplicity design style [J]. Yihai, 2013 